\title{
Italian Association of Sleep Medicine (AIMS) position statement and guideline on the treatment of menopausal sleep disorders
}

\author{
R. Silvestri ${ }^{\mathrm{a}, *}$, I. Aricò $^{\mathrm{a}}$, E. Bonanni ${ }^{\mathrm{b}}$, M. Bonsignore ${ }^{\mathrm{c}}$, M. Caretto $^{\mathrm{d}}$, D. Caruso ${ }^{\mathrm{e}}$, M.C. Di Perri ${ }^{\mathrm{a}}$, \\ S. Galletta ${ }^{\mathrm{a}}$, R.M. Lecca ${ }^{\mathrm{f}}$, C. Lombardi ${ }^{g, h}$, M. Maestri ${ }^{\mathrm{b}}$, M. Miccoli ${ }^{\mathrm{i}}$, L. Palagini ${ }^{\mathrm{e}}$, F. Provini ${ }^{\mathrm{j}, \mathrm{k}}$, \\ M. Puligheddu ${ }^{\text {f }}$ M. Savarese ${ }^{1}$, M.C. Spaggiari ${ }^{\mathrm{m}}$, T. Simoncini ${ }^{\mathrm{d}}$ \\ ${ }^{a}$ Center of Sleep Medicine, UOSD of Neurophysiopathology and Disorders of Movement, AOU G Martino, Department of Clinical and Experimental Medicine, University of \\ Messina, Italy \\ ${ }^{\mathrm{b}}$ Division of Neurology, Department of Clinical and Experimental Medicine, University of Pisa, Italy \\ ${ }^{\mathrm{c}}$ Division of Pneumology, University Hospital AOUP "Paolo Giaccone" PROMISE Department, University of Palermo, Italy \\ ${ }^{\mathrm{d}}$ Division of Obstetrics and Gynecology, Department of Clinical and Experimental Medicine, University of Pisa, Italy \\ ${ }^{\mathrm{e}}$ Department of Clinical and Experimental Medicine, Psychiatric Clinic, University of Pisa, Italy \\ ${ }_{\mathrm{f}}^{\mathrm{f}}$ Sleep Disorder Centre, Department of Medical Sciences and Public Health, University of Cagliari, Italy \\ ${ }^{g}$ Istituto Auxologico Italiano, IRCCS, Sleep Disorders Center \& Department of Cardiovascular, Neural and Metabolic Sciences, San Luca Hospital, Milan, Italy \\ ${ }^{\mathrm{h}}$ Department of Medicine and Surgery, University of Milano-Bicocca, Milan, Italy \\ ${ }^{\mathrm{i}}$ Department of Clinical and Experimental Medicine, University of Pisa, Italy \\ ${ }^{\mathrm{j}}$ IRCCS, Institute of Neurological Sciences, Bologna, Italy \\ ${ }^{\mathrm{k}}$ Department of BioMedical and NeuroMotor Sciences, University of Bologna, Italy \\ 1 "FM Puca" Neurology Unit, University Hospital Consortium Corporation Polyclinic of Bari, Italy \\ ${ }^{\mathrm{m}}$ Neurological Day Care Unit - Local Health Authority (AUSL 4), Parma, Italy
}

\section{A R T I C L E I N F O}

\section{Keywords:}

Menopause

Sleep disorders

Vasomotor symptoms (VMS)

Hormone replacement therapy (HRT)

Cognitive behavioral therapy for insomnia

(CBT-I)

Position statement

\begin{abstract}
A B S T R A C T
Insomnia, vasomotor symptoms (VMS) and depression often co-occur after the menopause, with consequent health problems and reductions in quality of life. The aim of this position statement is to provide evidence-based advice on the management of postmenopausal sleep disorders derived from a systematic review of the literature. The latter yielded results on VMS, insomnia, circadian rhythm disorders, obstructive sleep apnea (OSA) and restless leg syndrome (RLS). Overall, the studies show that menopausal hormone therapy (MHT) improves VMS, insomnia, and mood.

Several antidepressants can improve insomnia, either on their own or in association with MHT; these include selective serotonin reuptake inhibitors (SSRIs), serotonin and norepinephrine reuptake inhibitors (SNRIs), and mirtazapine. Long-term benefits for postmenopausal insomnia may also be achieved with non-drug strategies such as cognitive behavioral therapy (CBT) and aerobic exercise. Continuous positive airway pressure (CPAP) and mandibular advancement devices (MADs) both reduce blood pressure and cortisol levels in postmenopausal women suffering from OSA. However, the data regarding MHT on postmenopausal restless legs syndrome are conflicting.
\end{abstract}

\section{Introduction}

In women, aging and transition through menopause are accompanied by an increased prevalence of sleep disturbances. The Study of Women's Health Across the Nation (SWAN) [1] found that progression through the menopausal transition was associated with increasing selfreported sleep disturbances.

Sleep disturbances during the menopause include difficulty in sleep initiation or continuation, a higher frequency of night awakenings and poor-quality, non-restorative sleep. Sleep quality is worse in postmenopausal than in premenopausal women, with respect to both prolonged sleep latency (SL) and increased nocturnal time awake.

Other primary sleep disorders appear to increase during the periand postmenopausal years, including obstructive sleep apnea (OSA), with a prevalence of $45.2 \%$ for mild to moderate OSA and $10.1 \%$ for severe OSA (AHI > 30) [2], as well as sleep-related movement

\footnotetext{
* Corresponding author at: Center of Sleep Medicine, UOSD of Neurophysiopathology and Disorders of Movement, AOU G Martino, Department of Clinical and Experimental Medicine, University of Messina, Via Consolare Valeria, 1, 98124, Messina, ME, Italy.
}

E-mail address: rsilvestri@unime.it (R. Silvestri). 
disorders. Periodic leg movement disorders (PLMD) and restless legs syndrome (RLS) increase both in prevalence and in severity in the postmenopausal years [3].

Sleep disturbances during the menopause have been ascribed to several factors: physiological changes of aging, menopausal-related symptoms, stress, mood symptoms (e.g. depression and anxiety), and chronic health issues. Whether sleep problems during the menopausal years are the result of sleep being interrupted by vasomotor symptoms (VMS) or whether they are separate entities is still unclear.

Despite the importance of recognizing and treating sleep disorders in midlife women, relatively few studies have examined the effect of hormonal and non-hormonal treatments on sleep disorders and they have produced inconsistent findings. There is wide heterogeneity in the studies with respect to the various formulations used, treatment duration, the timing of treatment in relation to the menopause transition, and the inclusion and exclusion criteria of the clinical trials. Furthermore, there are no specific treatments for postmenopausal narcolepsy and other primary hypersomnias. The same is true for most parasomnias, with the exception of secondary enuresis and rapid eye movement (REM) behavior disorder (RBD), whose prevalence increases with age in both sexes, but again no therapy specifically targets postmenopausal women.

Finally, data concerning therapy are far from being uniform in relation to sleep-related movement disorders, such as PLMD and RLS, and nor do they cast any light on symptom aggravation [3].

This position statement and guideline on the treatment of postmenopausal sleep disorders by the Italian Association of Sleep Medicine (AIMS) was undertaken in 2017 by the Gender Sleep Medicine Committee, which acted as a task force. The position statement results from a systematic literature review (Table 1, Fig. 1).

\section{Methods}

All studies specifically addressing the treatment of postmenopausal sleep disorders from January 2000 until December 2018 were included in the review. Published studies were identified from the National Library of Medicine (Medline Database, Google Scholar and Scopus) by means of a search strategy using a combination of MESH terms and text combining the concepts of different sleep disorders and menopause. Postmenopause was defined as starting one year after the last spontaneous menstrual cycle. Included studies had to address the therapy of postmenopausal sleep disorders according to one of the following methodologies: case-series, case controls, cross-sectional studies, cohort studies, clinical trials (CTs), controlled clinical trials (CCTs), randomized controlled trials (RCTs) and systematic reviews (Fig. 1). Exclusion criteria included: language other than English, unavailable fulllength texts, dissertations, animal studies, correspondence, health letters, and case reports. After applying these criteria, 53 studies were taken into consideration. Among these, a maximum of 7 studies for each sleep disorder met the criteria. Table 1 summarizes all 23 RCTs discussed in the subsections below.

\section{Sleep disorders in the menopause}

In the following sections, we discuss the treatment of vasomotor symptoms, insomnia, circadian rhythm disorders, obstructive sleep apnea, and restless legs syndrome. Narcolepsy/ hypersomnia is not included due to the absence of studies in postmenopausal women.

\subsection{Sleep disorders, vasomotor symptoms, menopausal hormone therapy and non-hormonal options}

Estrogen-based therapy is known to be beneficial in treating menopause-related symptoms and improving the quality of life of postmenopausal women and is recommended in national and international guidelines [see for example 4,5]. Hormonal and non-hormonal pharmacological options will be discussed as well as non-drug therapies, as treatment needs to be individualized to a woman's needs and preferences $[4,[5]$.

Estrogen therapy with or without progesterone/ progestogen in non-hysterectomized women is very effective in treating VMS, thus indirectly improving sleep quality through a domino effect. By improving VMS, menopausal hormone therapy (MHT) improves sleep quality by decreasing the frequency of nighttime wakening, as shown in a study of women with severe hot flushes with objectively measured skin temperature and resistance and waking episodes [6]. A systematic review of MHT for the treatment of chronic insomnia during the menopause was published by Attarian et al. in 2015 [7]. They identified and reviewed 23 articles: 14 showed significant and continuous improvement in subjective sleep and VMS with low doses of estrogen, whereas the other 9 found no significant change in sleep parameters. In the same year Tansupswatdikul et al. [8] published a randomized, double-blind, placebo-controlled trial, using a $50 \mu \mathrm{g}$ transdermal estradiol patch which could raise estradiol levels to within the range of the follicular phase of the normal ovulatory cycle in postmenopausal women with sleep disorders but without severe VMS and/or recognized hot flushes during sleep. They found that estrogen therapy in insomniac postmenopausal women without severe vasomotor symptoms and/or recognized hot flushes during sleep did not improve sleep efficiency. Therefore, there are differences in response to MHT between women who have hot flushes during sleep and those who do not.

The tissue selective estrogen complex consisting of bazedoxifene/ conjugated estrogen (BZA/CE), has been evaluated in randomized, double-blind, placebo-controlled trials [9,10]. An initial 12-week study found that CE $0.45 \mathrm{mg} / \mathrm{BZA} 20 \mathrm{mg}$ was associated with a significant ( $\mathrm{p}<0.05$ ) improvement of sleep latency scores, sleep disturbance and sleep problem indices compared with placebo [9]. A subsequent oneyear randomized trial found that symptomatic postmenopausal women treated with BZA/CE demonstrated significant improvements in sleep and health-related quality of life, similar to women treated with CE/ medroxyprogesterone acetate (MPA) [10].

In addition, several studies show that MHT also alleviates depressive symptoms, which may also affect sleep in perimenopausal and postmenopausal women [11]. Thus, MHT is one of the recommended treatments for menopausal insomnia. It improves both quality of sleep and quality of life.

With regard to non-hormonal pharmacological treatments, some antidepressants and mood stabilizers (such as venlafaxine and gabapentin) can improve mood and VMS but may have negative effects on symptoms of insomnia. Several trials have shown that venlafaxine, a serotonin and norepinephrine reuptake inhibitor (SNRI), reduces VMS in healthy postmenopausal women and breast cancer survivors [see [12]]. Ensrud et al. [13] undertook a 3-arm double-blind randomized trial of low-dose estradiol, low-dose venlafaxine and placebo to study the effects on insomnia symptoms and sleep quality in peri- and postmenopausal women with VMS. The Insomnia Severity Index (ISI) and the Pittsburgh Sleep Quality Index (PSQI) were used to evaluate a patient's perception of sleep and to analyze subjective elements of insomnia. Women treated with low-dose estradiol compared with participants treated with placebo had a clinical improvement in subjective sleep quality, and reductions in both ISI and PSQI scores. The authors demonstrated a modest effect of treatment with venlafaxine in decreasing sleep disorders after 8 weeks of treatment. Low-dose venlafaxine and low-dose estradiol in healthy menopausal women with VMS were each more effective than placebo in reducing sleep disturbances and improving sleep quality. Other antidepressants such as escitalopram have also been found to improve hot flushes in randomized controlled trials [see for example 14].

Amongst all the non-pharmacological approaches, cognitive behavioral therapy (CBT) is the first-line treatment for chronic insomnia in adults of any age, as detailed below. Other interventions include highintensity exercise, hypnosis, yoga, and massage therapy [7]. Studies 


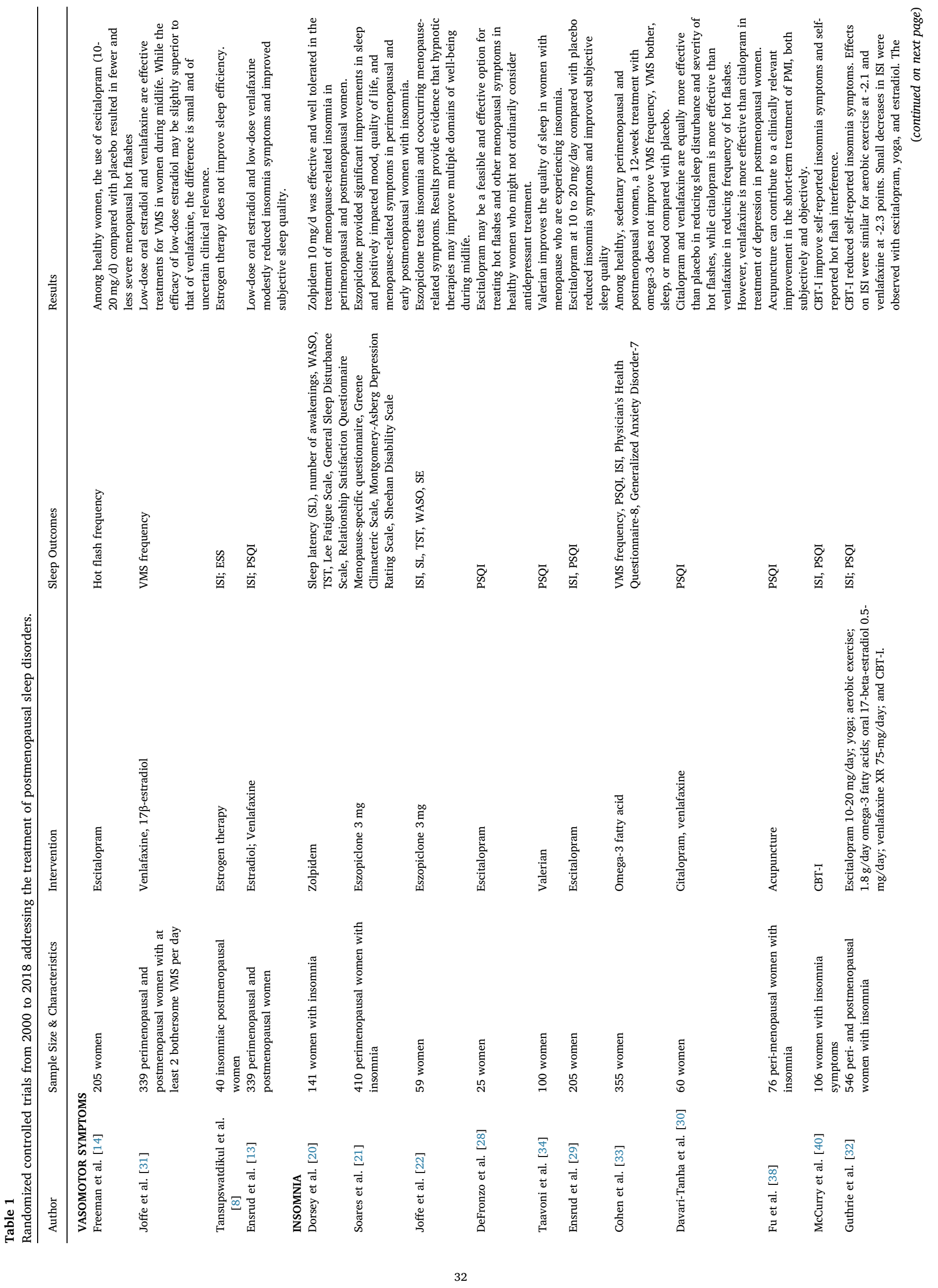




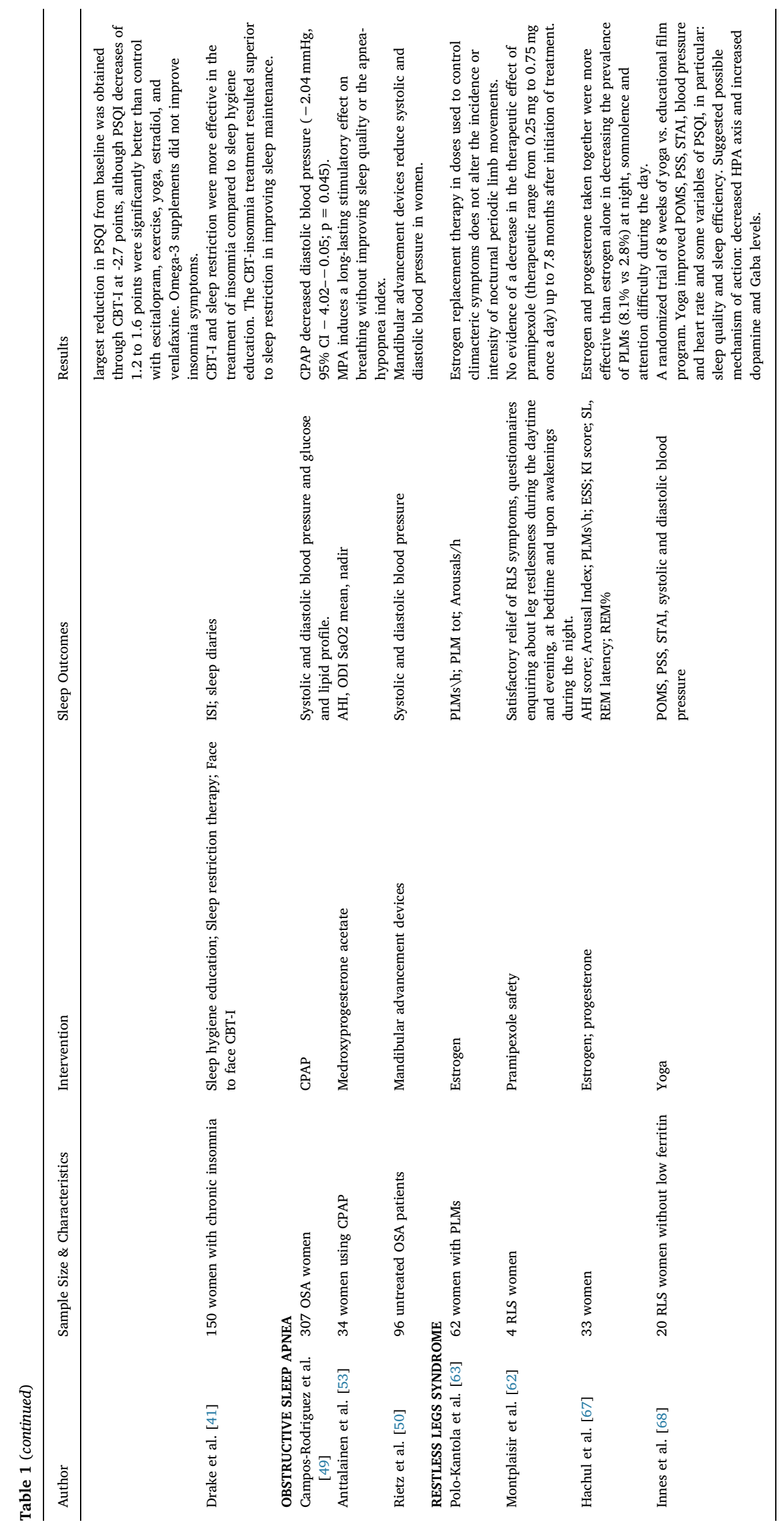




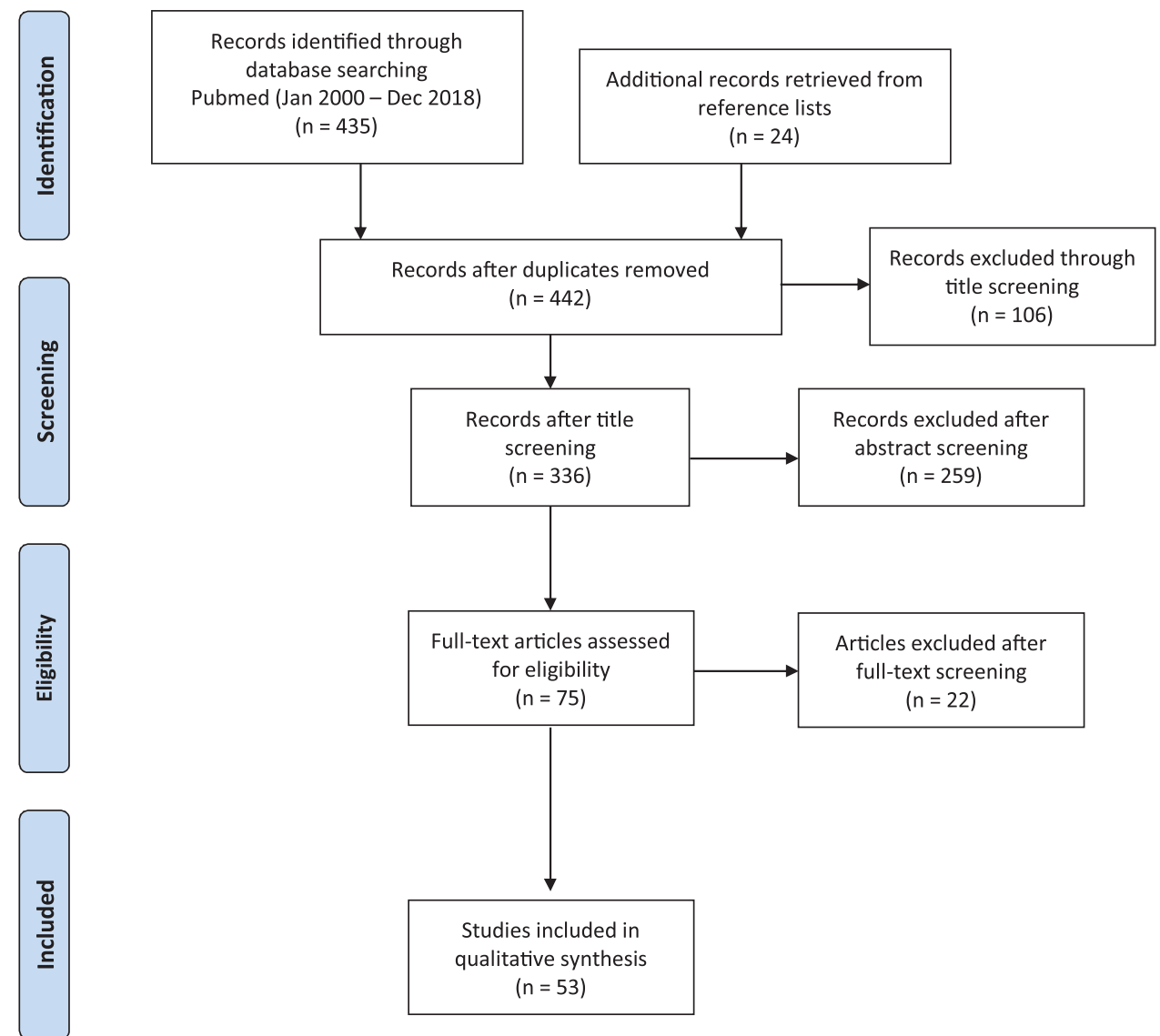

Fig. 1. Selection of Relevant Studies Regarding the Treatment of Postmenopausal Sleep Disorders. PRISMA flow chart of selection process.

evaluating the effects of aerobic training on menopausal symptoms and sleep quality are scant. Mansikkamäki and colleagues [15] enrolled 176 sedentary women, aged 43-63 years with menopausal symptoms (daily hot flushes), in a randomized controlled trial. Participants were randomized to a six-month unsupervised aerobic training intervention (50 min 4 times per week) or a control group. The exercise program of the intervention group consisted of aerobic training 4 times per week, with $50 \mathrm{~min}$ of exercise each time: women were to include at least two sessions of walking or Nordic walking (walking with poles or sticks, resembling cross-country skiing). At the end of study, women in the intervention group reported better sleep quality and a reduction in menopausal symptoms.

To conclude, hormonal and non-hormonal options have been found to be beneficial and choice of therapy needs to be personalized to individual women's comorbidities and preferences.

\subsection{Insomnia}

Management of insomnia during the menopausal transition constitutes a priority for the improvement of well-being because of its impact on daytime functioning, quality of life and health [16-19]. Treatments for insomnia during the menopause include sleep hygiene, behavioral interventions, and drugs, mainly hypnotics.

Few pharmacological studies have focused on this specific population, but these have shown positive results for hypnotics, antidepressants, melatonin, neuroleptics, and hormone therapy.

Non-benzodiazepine agonists of gamma-aminobutyric acid type A $\left(G_{A B A}\right)$ were the only drugs in this class to have been evaluated in randomized double-blind placebo studies. Zolpidem in 141 patients was effective after 4 weeks [20], while two studies reported the efficacy of eszopiclone at $3 \mathrm{mg}$ at 4 and 11 weeks, respectively, on 410 and 59 patients [21,22].
Ramelteon, an agonist of melatonin MT1 and MT2 receptors, has been approved by the US FDA for the treatment of chronic insomnia. A single open study of 20 menopausal women showed improvement in sleep at a nocturnal dose of $8 \mathrm{mg}$; that improvement became statistically significant at 4 weeks and even more pronounced at the sixth [23].

Few studies of antidepressants by menopausal women have considered sleep as an independent outcome measure. However, in a study of 600 menopausal women with moderate / severe VMS, paroxetine $7.5 \mathrm{mg}$ induced a significant reduction in associated awakenings and a significant increase in total sleep time [24]. Of note, paroxetine is approved for the management of VMS by the FDA but not in Europe. Among the other antidepressants, in a single study, mirtazapine $15 \mathrm{mg}$ was administered to 11 non-depressed perimenopausal women with chronic insomnia, for 4 weeks, followed by add-on prolonged-release melatonin (PRM) $2 \mathrm{mg}$ for 4 weeks with mirtazapine suspension at 1-3 months. The significant improvement in scores on the Pittsburgh Sleep Quality Index (PSQI) with mirtazapine persisted in the add-on phase and with PRM monotherapy [25]. A prospective, randomized, 9-month, placebo-controlled, double-blind study of citalopram and fluoxetine in the treatment of postmenopausal symptoms in 150 women found an improvement in insomnia only in the citalopram group [26].

Four studies have been conducted with escitalopram. An open-label study showed similar effects of escitalopram (10-20 mg) and estrogenbased therapy in improving sleep and VMS in a group of 32 depressed perimenopausal women [27]. A single-blind placebo-controlled study demonstrated an improvement in mood, sleep patterns, VMS, and overall quality of life with escitalopram $10-20 \mathrm{mg}$ in 25 women with VMS [28]. Furthermore, in the previously mentioned randomized trial of Freeman et al. [14], escitalopram improved insomnia symptoms and subjective sleep quality in healthy menopausal women with HF. Moreover, escitalopram 10-20 mg/day was effective and well tolerated for VMS, with improvement of subjective sleep quality and insomnia 
symptoms at 8-week follow-up [29].

A study by Davari-Tanha comparing citalopram, venlafaxine, and placebo with a sample of 60 patients concluded that while citalopram and venlafaxine were equally more effective than placebo in reducing sleep disturbance and severity of hot flushes, citalopram was more effective in reducing frequency of hot flushes than venlafaxine [30]. Furthermore, while venlafaxine seems to be slightly less effective than low-dosage oral estradiol on VMS, the difference is small and of uncertain clinical relevance [31].

The Health Network, involving 546 peri- and postmenopausal women with 'bothersome' HF (between 12 or 13 episodes per week), examined 6 types of interventions, using as outcome measures the Insomnia Severity Index (ISI) and the Pittsburgh Sleep Quality Index (PSQI) over 8 to 12 weeks of treatment [32]. Escitalopram 10-20 mg/ day; yoga; aerobics exercises; $1.8 \mathrm{~g} /$ day omega-3 fatty acids; oral 17beta-estradiol $0.5 \mathrm{mg}$ /day; venlafaxine XR $75 \mathrm{mg} /$ day; and cognitive behavioral therapy for insomnia (CBT-I) were evaluated. CBT-I proved to be the most effective intervention, followed by venlafaxine and exercise, then escitalopram. CBT-I produced the largest reduction in ISI score. The effects on ISI score were similar for exercise, at -2.1 points, and venlafaxine, at -2.3 points, and with escitalopram, yoga, and estradiol. The largest reduction in PSQI score from baseline was with CBT-I, at -2.7 points (from -3.9 to -1.5 ), although the reductions in PSQI score, from 1.2 to 1.6 , were significantly better than those achieved by escitalopram, exercise, yoga, estradiol, and venlafaxine. Omega-3 supplements did not improve the symptoms of insomnia, mood disorders or VMS [33], while in a single study of 100 postmenopausal women with insomnia, valerian seemed to improve the quality of sleep [34].

Some experimental data in animals and in humans support the hypothesis that menopausal estrogen deficiency could lead to a hyperactivity of the orexinergic system and contribute to anxiety, insomnia, and VMS [35]. It may therefore be hypothesized that orexin receptor antagonists represent a new non-hormonal strategy for menopause symptom management [36].

The initial cause of insomnia could be hormonal fluctuations and VMS such as night sweats, but physiological awakenings, behavioral conditioning, and maladaptive coping could act as perpetuating factors. CBT-I targets these perpetuating behaviors and has been effective in the treatment of chronic insomnia in randomized trials in adult and elderly women [37].

Other non-pharmacological options such as acupuncture [38], mindfulness, reflexology, exercise, and yoga have shown some positive results in small groups, but these have yet to be confirmed in larger studies. A short-term randomized trial has found a positive effect of acupuncture on perimenopausal insomnia [38].

\subsubsection{Cognitive behavioral therapy for insomnia (CBT-I)}

According to recent European guidelines, it is recommended that treatment of insomnia consists of psychological therapy, pharmacological therapy, or a combination of both [39]. First-line treatment for insomnia is considered to be cognitive behavioral therapy for insomnia (CBT-I), which is multimodal and consists of a combination of cognitive therapy, behavioral interventions, such as sleep restriction and stimulus control, and educational interventions, such as sleep hygiene [40].

Three clinical studies on CBT-I have been undertaken and are described below [Table 1].

Two studies belong to the Menopause Strategies Finding Lasting Answers for Symptoms and Health research network (MsFLASH). The trial conducted by McCurry et al. [40] was a single-site, randomized controlled trial of a telephone CBT-I intervention versus telephone-delivered menopause education over 8 weeks in 104 perimenopausal women with insomnia symptoms assessed with the Insomnia Severity Index scale (ISI) and sleep diaries. The CBT-I and menopause education interventions both consisted of six 20-30-minute telephone sessions over eight weeks. The CBT-I protocol provided information about age/ menopause-related sleep changes and the rationale for a behavioral approach, by re-scheduling sleep using sleep restriction and stimulus control instructions, changing beliefs/attitudes about sleep, and applying sleep hygiene recommendations. The menopausal education protocol included educational content and texts relevant to women's health and quality of life. Sessions were designed to reduce uncertainty about changes occurring during menopause and to help women identify symptom self-management strategies with no practice or instruction in CBT-I principles. This first randomized controlled trial showed that brief, telephone-delivered CBT-I resulted in significant 8- and 24-week improvements in self-reported insomnia symptoms, overall sleep quality, sleep latency, wake time after sleep onset, and sleep efficiency compared with the menopausal educational protocol. In addition, CBT-I was able to reduce self-reported HF interference with sleep at 8 and 24 weeks compared with the menopausal educational protocol. The authors hypothesized that the cognitive strategies taught during CBT-I may reduce daytime dysfunction associated with sleep loss dependent on VMS response.

A second study from MsFLASH [32] compared the results of several randomized control trials studying the effects of different interventions on insomnia and VMS. As previously seen, findings from these pooled analyses suggested that telephone-delivered CBT-I was more effective for reducing moderate to severe symptoms of insomnia in menopausal women with $\mathrm{HF}$ than other commonly used pharmacological or lifestyle modification options considered. In settings where CBT-I is not available, data have suggested that exercise and venlafaxine may produce moderate improvements in both self-reported insomnia symptoms and sleep quality ratings.

A recent controlled trial by Drake et al. [41] evaluated 150 postmenopausal women with chronic insomnia according to DSM-5 criteria and assessed with ISI and sleep diaries. Interventions were: sleep hygiene education, versus sleep restriction therapy, versus face-to-face CBT-I. Blinded assessments were performed at baseline, post-treatment, and 6 months after treatment. CBT-I and sleep restriction were more effective in the treatment of insomnia than sleep hygiene education. Although the responses to CBT-I and sleep restriction were similar, CBTI treatment exceeded sleep restriction in improving sleep maintenance, a common problem following the menopausal transition.

Taken together, all three studies demonstrate the efficacy of CBT-I in the treatment of menopausal insomnia, confirming it as a first-line treatment for these symptoms. Despite this, data are not statistically comparable.

\subsection{Circadian rhythm disorders}

Circadian misalignment has not been reported as a sleep disorder within the menopausal transition. No studies have specifically addressed circadian rhythm disorder therapy in postmenopausal women. The few studies undertaken have all found a decrease in melatonin levels through aging and in particular after menopause [42-44]. Furthermore, a study comparing healthy pre- and postmenopausal women found that postmenopausal women had lower nighttime serum melatonin concentrations than perimenopausal women. The duration of melatonin secretion tended to be shorter in the postmenopause whereas melatonin peak time did not differ. [45]. Furthermore, lower levels of melatonin in postmenopausal women may correlate with depressive symptoms [46]. Thus, melatonin therapy may have a favorable effect on those and related symptoms, such as VMS [47].

\subsection{Obstructive sleep apnea (OSA)}

Obstructive sleep apnea (OSA) is a common disorder characterized by loss of pharyngeal dilator muscle tone during sleep inducing recurrent upper airway collapse, leading to apnea and hypopnea. The obstructive events are associated with intermittent hypoxia/ hypercapnia, intrathoracic pressure changes and sleep arousal. These in turn unleash a series of cardiovascular and neurohumoral processes, 
including sympathetic nervous system hyperactivity, surges in heart rate and blood pressure, myocardial wall stress, oxidative stress, systemic inflammation, platelet aggregation, and impaired vascular endothelial function, which all contribute to increased cardiovascular risk [48]. It has therefore been suggested that OSA treatment could play a pivotal role in cardiovascular prevention strategies. However, there is little information on the effects of OSA treatment on cardiovascular outcomes in women, in particular after the menopause.

Additionally, the studies of the cardiovascular effects of OSA treatment in women have often produced conflicting results, probably due to their methodological limitations: studies are different in methodology, design, inclusion and exclusion criteria, outcomes, and treatment approaches.

One of the few available studies on this topic is that undertaken by Campos-Rodriguez et al. [49], who investigated the effect of CPAP on blood pressure and glucose and lipid profiles in women with moderate to severe OSA. This was a multicenter, open-label, randomized controlled 12-week trial analyzing the effect of CPAP on blood pressure and metabolic profile in 307 women aged 18-75 years, referred for suspicion of OSA and diagnosed with moderate to severe OSA (apnea-hypopnea index (AHI) $>15$ events/h). Women were randomized to CPAP $(n=151)$ or conservative treatment $(n=156)$ for 12 weeks. Compared with the control group, the CPAP group achieved a significantly greater decrease in diastolic blood pressure, and an insignificantly greater decrease in systolic blood pressure and mean blood pressure. However, CPAP therapy did not change any of the metabolic variables compared with conservative treatment. The greatest changes in blood pressure values were found in the group of women presenting excessive daytime sleepiness with high systolic blood pressure or diastolic blood pressure at baseline, and with severe OSA.

Positive effects of OSA treatment on blood pressure have been also described in a study by Rietz and coworkers [50]. That study was a 4month, double-blind, randomized controlled trial to determine the effect of mandibular advancement device (MAD) therapy versus sham devices on ambulatory nighttime and daytime blood pressure in women and men with daytime sleepiness and snoring or mild to moderate sleep apnea (apnea-hypopnea index $<30$ ). The study included 27 women and 58 men, aged 31-70 years. In women, MADs significantly lowered mean nighttime systolic blood pressure and mean nighttime adjusted diastolic blood pressure. In men, there were no significant differences in blood pressure at night or during the daytime between the intervention groups.

The effect of CPAP treatment on the hypothalamic-pituitary-adrenal axis was explored by Kritikou and coworkers [51] in a 2-month placebo-controlled trial exploring the effect of CPAP versus sham CPAP in 72 women and men. In both apneic men and women, OSA was associated with significantly higher 24-h cortisol levels compared with controls, whereas CPAP lowered cortisol levels significantly, close to those of controls.

On the other hand, Jennum et al. [52], who reported a registry study with 10 years of follow-up, found that CPAP therapy was associated with reduced all-cause mortality in middle-aged and elderly men, but no significant effect was found in women. They showed that women with OSA had lower mortality rates than men, irrespective of whether or not they received CPAP treatment. CPAP had no significant effect in 20- to 39-year-olds, but the overall mortality in this age group was small. Survival was increased by CPAP in 40- to 59-year-old and $>60$ year-old men, but no such effect was observed in women.

Finally, Anttalainen and coworkers [53] performed a placebo-controlled, double-blind, parallel-group trial to assess the effects of progestogen medroxyprogesterone acetate [MPA] in stimulating breathing and inducing sleep, and to compare MPA with CPAP. The study involved 34 postmenopausal women whose sleep disordered breathing had been treated with nasal CPAP for 6 months to 8 years prior to study entry. The 6-week trial included measurements with CPAP at baseline, after 14 days of placebo or MPA (60 mg daily), and after a 3-week washout. The study found that MPA induced a long-lasting stimulatory effect on breathing without improving sleep quality or the apnea-hypopnea index.

To conclude, both CPAP and MADs appear to lower blood pressure in women with OSA, with the greatest changes in those with severe disease, daytime sleepiness, and hypertension. On the other hand, CPAP adherence had no effect on mortality and MPA alone did not improve apnea-hypopnea index, nor sleep quality.

\subsection{Restless legs syndrome (RLS)}

RLS is a sensorimotor neurological disorder characterized by an urge to move the legs while resting, especially in the evening and at night. The prevalence of RLS increases with age, from about $0.5 \%$ for children to $5 \%$ for those older than70 years. In adults older than 40 years, RLS occurs about twice as often in women as in men [54]. Symptom severity increases after menopause [55,56].

Hachul et al. [57] reported worse RLS symptoms in the early compared with late postmenopause, despite more sleep complaints in the late postmenopausal group. However, no difference in polysomnography (PSG)-detected periodic leg movements (PLMs) was found between the two groups. Furthermore, a positive correlation between VMS and RLS symptoms was detected. In a later study [58], the same authors reported more RLS subjective complaints in precompared with postmenopausal women, whereas the PLMs index was significantly higher in the postmenopausal group. A more recent study [59] undertaken in 980 Iranian postmenopausal women found that the prevalence of RLS was $16.02 \%$. The risk was increased in women with low socio-economic status, smokers and higher body mass index, and reduced in MHT users.

A Swedish study reported increased prevalence of RLS among women with VMS during the menopause transition, but not in MHT users [60]. In a subsequent study [61], the same authors noted an association between women with VMS and clinically significant periodic limb movements (PLMs)/RLS, but not PLMs with arousal. The mechanisms underlying the association are unclear.

With regard to treatment Montplaisir et al. [62] report a positive long-term effect of pramipexole ( $0.25-0.75 \mathrm{mg}$ ) on RLS severity in seven patients, of whom 4 were women, mean age 55.4 years, evaluated after 1 month and after 7.8 months of therapy.

While MHT is an effective treatment for VMS [4,5], benefit has not been found in RLS [63-67]. Indeed, MHT trials on RLS/PLMs mostly suggest a negative or unmodified outcome, as reported by an extensive review [3] that cites, among others, two particularly noteworthy studies. Of the latter, a randomized double-blind placebo-controlled crossover study [63] conducted with 62 postmenopausal women reporting no RLS, but some PLMs (48\%), showed that oral estradiol $(2.5 \mathrm{mg} /$ day $)$ or estradiol hemihydrate $(50 \mu \mathrm{gs} / 24 \mathrm{~h})$ patches did not significantly affect PLMs compared with placebo (31\% vs. $27 \%$, respectively).

Similarly, Montplaisir et al. [64], in a study of 21 postmenopausal women with subjective complaints of poor nocturnal sleep but no history of RLS, found no significant difference in PLMs after six months of MHT consisting of (1) estrogen (Premarin $0.625 \mathrm{mg}$ ) and medroxyprogesterone acetate (Provera $5 \mathrm{mg})(\mathrm{n}=11)$ or $(2)$ estrogen (Premarin $0.625 \mathrm{mg}$ ) and oral micronized progesterone (Prometrium $200 \mathrm{mg}$ ).

A subsequent review paper [65] suggested that the situation is complex in postmenopausal women with both VMS and RLS, especially with cyclical progestogen addition. However, a case report described a decrease in PLMs in a postmenopausal woman given transdermal estradiol [66]. The same authors [67] subsequently undertook a randomized placebo-controlled study of MHT on sleep in 33 postmenopausal women. The women were initially given estrogen alone or placebo for 12 weeks. Then, while still taking estrogen or placebo, they all received additional medroxyprogesterone acetate $5 \mathrm{mg}$ daily for another 12 
weeks. They found that estrogen plus medroxyprogesterone acetate was more effective than estrogen alone in decreasing the prevalence of PLMs and hot flashes.

Hence, in light of these studies, we agree with Manconi et al. [3] that the available data with regard to MHT are insufficient to endorse its use as a viable approach to postmenopausal RLS.

Finally, a preliminary randomized trial [68] reported beneficial effects of an 8-week gentle yoga program in 20 postmenopausal women as well as pilot study in 13 women [69]. Further data are awaited.

\section{Summary and recommendations}

- Menopausal sleep disorders have a wide variety of manifestations, including not only insomnia but also breathing and movement disorders. VMS have a large effect on postmenopausal women's sleep, mood, and quality of life. Treatment needs to be individualized, taking into account comorbidities and preferences.

- Insomnia per se best responds to CBT-I, MHT, and escitalopram. New drugs with different therapeutic targets are in development.

- Melatonin may stabilize circadian rhythm while helping insomnia symptoms as well as VMS.

- CPAP and MADs decrease the hypertension burden of OSA, but without a major impact on sleep quality and mortality risk.

- The data regarding MHT on RLS and PLMs are conflicting. MHT may have a preventive role rather than being a treatment option.

- There is a need for adequately powered randomized controlled trials as well as cohort studies to better understand the impact of menopausal sleep disorders and increase the evidence-base of therapeutic strategies.

\section{Contributors}

Rosalia Silvestri contributed to conceptualization, investigation, validation, formal analysis, writing, review and editing of the draft, supervision, and project administration.

Irene Aricò contributed to investigation.

Enrica Bonanni contributed to investigation, and writing, review and editing of the draft.

Marisa Bonsignore contributed to investigation and writing the draft.

Marta Caretto contributed to investigation, and writing, review and editing of the draft.

Danila Caruso contributed to writing the draft.

Maria Caterina Di Perri contributed to methodology, investigation, and writing, review and editing of the draft.

Santi Galletta contributed to methodology, review and editing of the draft, and data curation.

Rosa Maria Lecca contributed to investigation.

Carolina Lombardi contributed to investigation and writing the draft.

Michelangelo Maestri contributed to investigation and writing the draft.

Mario Miccoli contributed to methodology, formal analysis, data curation, visualization, and writing the draft.

Laura Palagini contributed to investigation, and writing, review and editing of the draft.

Federica Provini contributed to investigation, conceptualization, and writing, review and editing of the draft.

Monica Puligheddu contributed to investigation.

Mariantonietta Savarese contributed to investigation.

Maria Cristina Spaggiari contributed to investigation.

Tommaso Simoncini contributed to conceptualization, investigation, validation, supervision, and project administration.

\section{Declaration of Competing Interest}

The authors declare that they have no conflict of interest.

\section{Funding}

This research did not receive any specific grant from funding agencies in the public, commercial, or not-for-profit sectors.

\section{Provenance and peer review}

AIMS position statement and guideline, not externally peer reviewed.

\section{References}

[1] H.M. Kravitz, X. Zhao, J.T. Bromberger, E.B. Gold, M.H. Hall, K.A. Matthews, M.R. Sowers, Sleep disturbance during the menopausal transition in a multi-ethnic community sample of women, Sleep. 31 (2008) 979-990.

[2] R. Heinzer, H. Marti-Soler, P. Marques-Vidal, N. Tobback, D. Andries, G. Waeber, M. Preisig, P. Vollenweider, J. Haba-Rubio, Impact of sex and menopausal status on the prevalence, clinical presentation, and comorbidities of sleep-disordered breathing, Sleep Med. 51 (2018) 29-36.

[3] M. Manconi, J. Ulfberg, K. Berger, I. Ghorayeb, J. Wesström, S. Fulda, R.P. Allen, T. Pollmächer, When gender matters: restless legs syndrome. Report of the "RLS and woman" workshop endorsed by the European RLS study group, Sleep Med. Rev. 16 (2012) 297-307.

[4] E. Armeni, I. Lambrinoudaki, I. Ceausu, H. Depypere, A. Mueck, F.R. Pérez-López, Y.T. Schouw, L.M. Senturk, T. Simoncini, J.C. Stevenson, P. Stute, M. Rees, Maintaining postreproductive health: a care pathway from the European Menopause and Andropause Society (EMAS), Maturitas 89 (2016) 63-72.

[5] The National Institute for Health and Care Excellence, Menopause: diagnosis and management NICE guideline [NG23] Published date, 2015 November Accessed 12 August 2019 https://www.nice.org.uk/guidance/ng23.

[6] Y. Erlik, I.V. Tataryn, D.R. Meldrum, H.L. Judd, P. Lomax, J.G. Bajorek, Association of waking episodes with menopausal hot flushes, JAMA J. Am. Med. Assoc. 245 (1981) 1741-1744.

[7] H. Attarian, H. Hachul, T. Guttuso, B. Phillips, Treatment of chronic insomnia disorder in menopause: evaluation of literature, Menopause. 22 (2015) 674-684.

[8] P. Tansupswatdikul, S. Chaikittisilpa, N. Jaimchariyatam, K. Panyakhamlerd, U. Jaisamrarn, N. Taechakraichana, Effects of estrogen therapy on postmenopausal sleep quality regardless of vasomotor symptoms: A randomized trial, Climacteric 18 (2015) 198-204.

[9] W. Utian, H. Yu, J. Bobula, S. Mirkin, S. Olivier, J.H. Pickar, Bazedoxifene/conjugated estrogens and quality of life in postmenopausal women, Maturitas 63 (2009) 329-335.

[10] J.V. Pinkerton, K. Pan, L. Abraham, J. Racketa, K.A. Ryan, A.A. Chines, S. Mirkin, Sleep parameters and health-related quality of life with bazedoxifene/conjugated estrogens: a randomized trial, Menopause 21 (2014) 252-259.

[11] P.M. Maki, S.G. Kornstein, H. Joffe, J.T. Bromberger, E.W. Freeman, G. Athappilly, W.V. Bobo, L.H. Rubin, H.K. Koleva, L.S. Cohen, C.N. Soares, Guidelines for the evaluation and treatment of perimenopausal depression: summary and recommendations, J. Womens Health (Larchmt) 28 (February 2) (2019) 117-134.

[12] A.P. Handley, M. Williams, The efficacy and tolerability of SSRI/SNRIs in the treatment of vasomotor symptoms in menopausal women: a systematic review, J. Am. Assoc. Nurse Pract. 27 (January 1) (2015) 54-61.

[13] K.E. Ensrud, K.A. Guthrie, C. Hohensee, B. Caan, J.S. Carpenter, E.W. Freeman, A.Z. LaCroix, C.A. Landis, J. Manson, K.M. Newton, J. Otte, S.D. Reed, J.L. Shifren, B. Sternfeld, N.F. Woods, H. Joffe, Effects of estradiol and venlafaxine on insomnia symptoms and sleep quality in women with hot flashes, Sleep 38 (2015) 97-108.

[14] E.W. Freeman, K.A. Guthrie, B. Caan, B. Sternfeld, L.S. Cohen, H. Joffe, J.S. Carpenter, G.L. Anderson, J.C. Larson, K.E. Ensrud, S.D. Reed, K.M. Newton, S. Sherman, M.D. Sammel, A.Z. LaCroix, Efficacy of escitalopram for hot flashes in healthy menopausal women: a randomized controlled trial, JAMA - J. Am. Med. Assoc. 305 (2011) 267-274.

[15] K. Mansikkamäki, J. Raitanen, C.H. Nygård, R. Heinonen, T. Mikkola, E. Tomás R. Luoto, Sleep quality and aerobic training among menopausal women - A randomized controlled trial, Maturitas. 72 (2012) 339-345.

[16] F.C. Baker, A.R. Willoughby, S.A. Sassoon, I.M. Colrain, M. de Zambotti, Insomnia in women approaching menopause: beyond perception, Psychoneuroendocrinology. 60 (2015) 96-104.

[17] F.C. Baker, M. De Zambotti, I.M. Colrain, B. Bei, Sleep problems during the menopausal transition: Prevalence, impact, and management challenges, Nat. Sci. Sleep 10 (2018) 73-95.

[18] J.E. Blümel, A. Cano, E. Mezones-Holguín, G. Barón, A. Bencosme, Z. Benítez, L.M. Bravo, A. Calle, D. Flores, M.T. Espinoza, G. Gómez, J.A. Hernández-Bueno, F. Laribezcoa, M. Martino, S. Lima, A. Monterrosa, D. Mostajo, E. Ojeda, W. Onatra, H. Sánchez, K. Tserotas, M.S. Vallejo, S. Witis, M.C. Zúñiga, P. Chedraui, A multinational study of sleep disorders during female mid-life, Maturitas. 72 (2012) 359-366.

[19] Q. Xu, C.P. Lang, Examining the relationship between subjective sleep disturbance 
and menopause: a systematic review and meta-analysis, Menopause 21 (2014) $1301-1318$

[20] C.M. Dorsey, K.A. Lee, M.B. Scharf, Effect of Zolpidem on sleep in women with perimenopausal and postmenopausal insomnia: A 4-week, randomized, multicenter, double-blind, placebo-controlled study, Clin. Ther. 26 (2004) 1578-1586.

[21] C.N. Soares, H. Joffe, R. Rubens, J. Caron, T. Roth, L. Cohen, Eszopiclone in patients with insomnia during perimenopause and early postmenopause: a randomized controlled trial, Obstet. Gynecol. 108 (2006) 1402-1410.

[22] H. Joffe, L. Petrillo, A. Viguera, A. Koukopoulos, K. Silver-Heilman, A. Farrell, G. Yu, M. Silver, L.S. Cohen, Eszopiclone improves insomnia and depressive and anxious symptoms in perimenopausal and postmenopausal women with hot flashes: a randomized, double-blinded, placebo-controlled crossover trial, Am. J. Obstet. Gynecol. 202 (2010) 171.e1-171.e11.

[23] R.D. Dobkin, M. Menza, K.L. Bienfait, L.A. Allen, H. Marin, M.A. Gara, Ramelteon for the treatment of insomnia in menopausal women, Menopause Int. 15 (2009) 13-18.

[24] J.V. Pinkerton, H. Joffe, K. Kazempour, H. Mekonnen, S. Bhaskar, J. Lippman, Lowdose paroxetine $(7.5 \mathrm{mg})$ improves sleep in women with vasomotor symptoms associated with menopause, Menopause. 22 (2015) 50-58.

[25] Z. Dolev, Case series of perimenopausal women with insomnia treated with mirtazapine followed by prolonged-release melatonin add-on and monotherapy, Arch. Womens Ment. Health 14 (2011) 269-273.

[26] E. Suvanto-Luukkonen, R. Koivunen, H. Sundström, R. Bloigu, E. Karjalainen, L. Häivä-Mällinen, J.S. Tapanainen, Citalopram and fluoxetine in the treatment of postmenopausal symptoms: a prospective, randomized, 9-month, placebo-controlled, double-blind study, Menopause. 12 (2005) 18-26.

[27] C.N. Soares, H. Arsenio, H. Joffe, B. Bankier, P. Cassano, L.F. Petrillo, L.S. Cohen, Escitalopram versus ethinyl estradiol and norethindrone acetate for symptomatic peri- and postmenopausal women: impact on depression, vasomotor symptoms, sleep, and quality of life, Menopause 13 (2006) 780-786.

[28] D.R. DeFronzo, M. Matthew, A.L. A, M. Humberto, B.K. L, T. Jade, H. Jennifer, Escitalopram reduces hot flashes in nondepressed menopausal women: a pilot study, Ann. Clin. Psychiatry 21 (2009) 70-76.

[29] K.E. Ensrud, H. Joffe, K.A. Guthrie, J.C. Larson, S.D. Reed, K.M. Newton, B. Sternfeld, A.Z. Lacroix, C.A. Landis, N.F. Woods, E.W. Freeman, Effect of escitalopram on insomnia symptoms and subjective sleep quality in healthy perimenopausal and postmenopausal women with hot flashes: a randomized controlled trial, Menopause. 19 (2012) 848-855.

[30] F. Davari-Tanha, M. Soleymani-Farsani, M. Asadi, M. Shariat, M. Shirazi, H. Hadizadeh, Comparison of citalopram and venlafaxine's role in treating sleep disturbances in menopausal women, a randomized, double-blind, placebo-controlled trial, Arch. Gynecol. Obstet. 293 (2016) 1007-1013.

[31] H. Joffe, K.A. Guthrie, A.Z. LaCroix, S.D. Reed, K.E. Ensrud, J.A.E. Manson, K.M. Newton, E.W. Freeman, G.L. Anderson, J.C. Larson, J. Hunt, J. Shifren, K.M. Rexrode, B. Caan, B. Sternfeld, J.S. Carpenter, L. Cohen, Low-dose estradiol and the serotonin-norepinephrine reuptake inhibitor venlafaxine for vasomotor symptoms: a randomized clinical trial, JAMA Intern. Med. 174 (2014) 1058-1066.

[32] K.A. Guthrie, J.C. Larson, K.E. Ensrud, G.L. Anderson, J.S. Carpenter, E.W. Freeman, H. Joffe, A.Z. LaCroix, J.A.E. Manson, C.M. Morin, K.M. Newton, J. Otte, S.D. Reed, S.M. McCurry, Effects of pharmacologic and nonpharmacologic interventions on insomnia symptoms and self-reported sleep quality in women with hot flashes: a pooled analysis of individual participant data from four MsFLASH trials, Sleep 41 (January 1) (2018), https://doi.org/10.1093/sleep/zsx190.

[33] L.S. Cohen, H. Joffe, K.A. Guthrie, K.E. Ensrud, M. Freeman, J.S. Carpenter, L.A. Learman, K.M. Newton, S.D. Reed, J.E. Manson, B. Sternfeld, B. Caan, E.W. Freeman, A.Z. Lacroix, L.F. Tinker, C. Booth-Laforce, J.C. Larson, G.L. Anderson, Efficacy of omega-3 for vasomotor symptoms treatment: A randomized controlled trial, Menopause 21 (2014) 347-354.

[34] S. Taavoni, N. Ekbatani, M. Kashaniyan, H. Haghani, Effect of valerian on sleep quality in postmenopausal women: a randomized placebo-controlled clinical trial, Menopause 18 (2011) 951-955.

[35] L.M. Federici, I.F. Caliman, A.I. Molosh, S.D. Fitz, W.A. Truitt, P. Bonaventure, J.S. Carpenter, A. Shekhar, P.L. Johnson, Hypothalamic orexin's role in exacerbated cutaneous vasodilation responses to an anxiogenic stimulus in a surgical menopause model, Psychoneuroendocrinology 65 (2016) 127-137.

[36] D. Cintron, J.P. Beckman, K.R. Bailey, B.D. Lahr, M. Jayachandran, V.M. Miller, Plasma orexin A levels in recently menopausal women during and 3 years following use of hormone therapy, Maturitas 99 (2017) 59-65.

[37] J.M. Trauer, M.Y. Qian, J.S. Doyle, S.M.W. Rajaratnam, D. Cunnington, Cognitive behavioral therapy for chronic insomnia: a systematic review and meta-analysis, Ann. Intern. Med. 163 (2015) 191-204.

[38] C. Fu, N. Zhao, Z. Liu, L.H. Yuan, C. Xie, W.J. Yang, X.T. Yu, H. Yu, Y.F. Chen, Acupuncture improves peri-menopausal insomnia: a randomized controlled trial, Sleep 40 (2017) 11, https://doi.org/10.1093/sleep/zsx153.

[39] D. Riemann, C. Baglioni, C. Bassetti, B. Bjorvatn, L. Dolenc Groselj, J.G. Ellis, C.A. Espie, D. Garcia-Borreguero, M. Gjerstad, M. Gonçalves, E. Hertenstein, M. Jansson-Fröjmark, P.J. Jennum, D. Leger, C. Nissen, L. Parrino, T. Paunio, D. Pevernagie, J. Verbraecken, H.G. Weeß, A. Wichniak, I. Zavalko, E.S. Arnardottir, O.C. Deleanu, B. Strazisar, M. Zoetmulder, K. Spiegelhalder, European guideline for the diagnosis and treatment of insomnia, J. Sleep Res. 26 (2017) 675-700.

[40] S.M. McCurry, K.A. Guthrie, C.M. Morin, N.F. Woods, C.A. Landis, K.E. Ensrud, J.C. Larson, H. Joffe, L.S. Cohen, J.R. Hunt, K.M. Newton, J.L. Otte, S.D. Reed, B. Sternfeld, L.F. Tinker, A.Z. Lacroix, Telephone-Based cognitive behavioral therapy for insomnia in perimenopausal and postmenopausal women with vasomotor symptoms A MsFLASH randomized clinical trial, JAMA Intern. Med. 176
(2016) 913-920.

[41] C.L. Drake, D.A. Kalmbach, J.T. Arnedt, P. Cheng, C.V. Tonnu, A. CuamatziCastelan, C. Fellman-Couture, Treating chronic insomnia in postmenopausal women: A randomized clinical trial comparing cognitive-behavioral therapy for insomnia, sleep restriction therapy, and sleep hygiene education, Sleep. 42 (2019), https://doi.org/10.1093/sleep/zsy217.

[42] J.F. Walters, S.M. Hampton, G.A.A. Ferns, D.J. Skene, Effect of menopause on melatonin and alertness rhythms investigated in constant routine conditions, Chronobiol. Int. 22 (2005) 859-872.

[43] Y. Okatani, N. Morioka, A. Wakatsuki, Changes in nocturnal melatonin secretion in perimenopausal women: correlation with endogenous estrogen concentrations, J. Pineal Res. 28 (2000) 111-118.

[44] C. Gómez-Santos, C.B. Saura, J.A.R. Lucas, P. Castell, J.A. Madrid, M. Garaulet, Menopause status is associated with circadian- and sleep-related alterations, Menopause 23 (2016) 682-690.

[45] E. Toffol, N. Kalleinen, J. Haukka, O. Vakkuri, T. Partonen, P. Polo-Kantola, Melatonin in perimenopausal and postmenopausal women: associations with mood, sleep, climacteric symptoms, and quality of life, Menopause 21 (2014) 493-500.

[46] B.L. Parry, L. Fernando Martínez, E.L. Maurer, A.M. López, D. Sorenson, C.J. Meliska, Sleep, rhythms and women's mood. Part II. Menopause, Sleep Med. Rev. 10 (2006) 197-208.

[47] A.Y. Gursoy, M. Kiseli, G.S. Caglar, Melatonin in aging women, Climacteric 18 (2015) 790-796.

[48] P. Lévy, M. Kohler, W.T. McNicholas, F. Barbé, R.D. McEvoy, V.K. Somers, L. Lavie, J.L. Pépin, Obstructive sleep apnoea syndrome, Nat. Rev. Dis. Primers 25 (June 1) (2015) 15015 .

[49] F. Campos-Rodriguez, M. Gonzalez-Martinez, A. Sanchez-Armengol, B. JuradoGamez, J. Cordero-Guevara, N. Reyes-Nuñez, M.F. Troncoso, A. Abad-Fernandez, J. Teran-Santos, J. Caballero-Rodriguez, M. Martin-Romero, A. Encabo-Motiño, L. Sacristan-Bou, J. Navarro-Esteva, M. Somoza-Gonzalez, J.F. Masa, M.A. SanchezQuiroga, B. Jara-Chinarro, B. Orosa-Bertol, M.A. Martinez-Garcia, Spanish Sleep Network. Effect of continuous positive airway pressure on blood pressure and metabolic profile in women with sleep apnoea, Eur. Respir. J. 50 (August 2) (2017) pii: 1700257.

[50] H. Rietz, K.A. Franklin, B. Carlberg, C. Sahlin, M. Marklund, Nocturnal blood pressure is reduced by a mandibular advancement device for sleep apnea in women: findings from secondary analyses of a randomized trial, J. Am. Heart Assoc. 7 (2018).

[51] I. Kritikou, M. Basta, A.N. Vgontzas, S. Pejovic, J. Fernandez-Mendoza, D. Liao, E.O. Bixler, J. Gaines, G.P. Chrousos, Sleep apnoea and the hypothalamic-pituitaryadrenal axis in men and women: effects of continuous positive airway pressure, Eur. Respir. J. 47 (2016) 531-540.

[52] P. Jennum, P. Tønnesen, R. Ibsen, J. Kjellberg, All-cause mortality from obstructive sleep apnea in male and female patients with and without continuous positive airway pressure treatment: a registry study with 10 years of follow-up, Nat. Sci. Sleep 7 (2015) 43-50.

[53] U. Anttalainen, T. Saaresranta, T. Vahlberg, O. Polo, Short-term medroxyprogesterone acetate in postmenopausal women with sleep-disordered breathing: a placebo-controlled, randomized, double-blind, parallel-group study, Menopause. 21 (2014) 361-368.

[54] M.H. Kryger, T. Roth, W.C. Dement, Principles and Practice of Sleep Medicine, 5th ed., (2017).

[55] I. Ghorayeb, B. Bioulac, C. Scribans, F. Tison, Perceived severity of restless legs syndrome across the female life cycle, Sleep Med. 9 (2008) 799-802.

[56] M. Fabbrini, I. Aricò, F. Tramonti, R. Condurso, L. Carnicelli, A. De Rosa, C. Di Perri, M.R. Bonsignore, A. Zito, G. Russo, M.G. Pagliarulo, B. Guarnieri, G. Cerroni, G. Mennuni, G. Della Marca, E. Bonanni, R. Silvestri, Sleep disorders in menopause: results from an Italian multicentric study, Arch. Ital. Biol. 153 (2015), https://doi. org/10.12871/0003982920152345.

[57] H. Hachul, L.R.A. Bittencourt, J.M. Soares, S. Tufik, E.C. Baracat, Sleep in postmenopausal women: differences between early and late post-menopause, Eur. J. Obstet. Gynecol. Reprod. Biol. 145 (2009) 81-84.

[58] H. Hachul, M.L. Andersen, L.R.A. Bittencourt, R. Santos-Silva, S.G. Conway, S. Tufik, Does the reproductive cycle influence sleep patterns in women with sleep complaints? Climacteric. 13 (2010) 594-603.

[59] R. Bagheri, P. Abedi, P. Mousavi, N. Azimi, The prevalence of restless legs syndrome and its relationship with demographic characteristics and medical disorders in postmenopausal Iranian women, Health Care Women Int. (2018) 1-9.

[60] J. Wesström, S. Nilsson, I. Sundström-Poromaa, J. Ulfberg, Restless legs syndrome among women: prevalence, co-morbidity and possible relationship to menopause, Climacteric 11 (2008) 422-428.

[61] J. Wesström, J. Ulfberg, I. Sundström-Poromaa, E. Lindberg, Periodic limb movements are associated with vasomotor symptoms, J. Clin. Sleep Med. 10 (2014) $15-20$.

[62] J. Montplaisir, R. Denesle, D. Petit, Pramipexole in the treatment of restless legs syndrome: a follow-up study, Eur. J. Neurol. (2000) 27-31.

[63] P. Polo-Kantola, E. Rauhala, R. Erkkola, K. Irjala, O. Polo, Estrogen replacement therapy and nocturnal periodic limb movements: a randomized controlled trial, Obstet. Gynecol. 97 (2001) 548-554.

[64] J. Montplaisir, J. Lorrain, R. Denesle, D. Petit, Sleep in menopause: differential effects of two forms of hormone replacement therapy, Menopause. 8 (2001) 10-16.

[65] P.S. Eichling, J. Sahni, Menopause related sleep disorders, J. Clin. Sleep Med. 1 (2005) 291-300.

[66] H. Hachul, E.C. Baracat, J.M. Soares, M.A. Haidar, M.T. de Mello, S. Tufik, L.R.A. Bittencourt, Estrogen therapy reduces nocturnal periodic limb movements, Maturitas. 58 (2007) 319-322. 
[67] H. Hachul, L.R.A. Bittencourt, M.L. Andersen, M.A. Haidar, E.C. Baracat, S. Tufik, Effects of hormone therapy with estrogen and/or progesterone on sleep pattern in postmenopausal women, Int. J. Gynecol. Obstet. 103 (2008) 207-212.

[68] K.E. Innes, T.K. Selfe, The effects of a gentle yoga program on sleep, mood, and blood pressure in older women with restless legs syndrome (RLS): a preliminary randomized controlled trial, Evidence-Based Complement, Altern. Med. 2012 (2012).

[69] K.E. Innes, T.K. Selfe, P. Agarwal, K. Williams, K.L. Flack, Efficacy of an eight-week yoga intervention on symptoms of restless legs syndrome (RLS): a pilot study, J. Altern. Complement. Med. 19 (June 6) (2013) 527-535. 